Journal of

Accident and

Emergency

Medicine 1994

11, 231-233

\title{
Patients who attend two accident and emergency departments
}

\author{
H.R. GULY ${ }^{1}$ \& I.C. GRANT ${ }^{2}$ \\ ${ }^{1}$ Derriford Hospital, Derriford Road, Plymouth and ${ }^{2}$ Royal Naval Hospital, Clarence Place, \\ Plymouth
}

\section{SUMMARY}

Forty-six patients were identified who attended an accident and emergency (A\&E) department having previously attended a different A\&E department in the same city for the same problem. Of these patients, $20 \%$ had been referred to the second department by the general practitioner (GP) or practice nurse.

A diagnostic error had been made in $17.5 \%$ of patients at their first visit and some management errors were discovered. Eleven per cent of patients had an unnecessary second set of radiographs taken.

$A$ review of unplanned reattenders to an $A \& E$ department is an important opportunity for audit and normally an error will not be discovered if a patient attends a different department. We recommend that in cities where there is more than one A\&E department a system should be set up whereby if a patient attends one department having previously attended another, the first department should be informed of the patients reattendance, especially if any diagnostic or management error has been discovered. Patients should be educated that if they have sought medical help for any problem and the condition does not improve, then they should return to see the same doctor or A\&E department for continuity of care and avoidance of unnecessary duplication of investigations including exposure to X-rays.

Key words: accident and emergency department, audit, return visit

Correspondence: Henry R. Guly, Consultant in Accident and Emergency Medicine, Derriford Hospital, Derriford Road, Plymouth, Devon PL6 8DH, UK back. Patients who return to the A\&E department have previously been investigated and a percentage of them are found to have an injury which has been missed or to have been mis-managed at the initial visit. ${ }^{1}$ Most patients, however, return because they have unrealistic expectations of the duration of symptoms and better patient education at the initial visit should be able to prevent many reattendances. ${ }^{2}$ It is clear that to review patients who reattend A\&E departments is an important opportunity for clinical audit. $^{3}$

In cities with more than one A\&E Department, patients may return to a different A\&E department. This study looks at patients who attended more than one A\&E department in a city, for the same problem.

\section{METHODS}

At the time of study there were two A\&E departments in Plymouth. These were large A\&E departments at Derriford Hospital on the outskirts of the city, a district general hospital with subregional specialties and a smaller department in the city centre at the Royal Naval Hospital Plymouth. This was run by the Ministry of Defence and provided care for servicemen and their families and for any civilians who attended. It had a small catchment area for civilian ambulance patients and traditionally treated ex-servicemen and the population of the inner-city area surrounding the hospital.

Over a 4-month period note was made of all patients who attended either of the two departments who said that they had previously attended the other department for the same problem. Patients were asked why they had attended the second department and the answer was noted, but doctors and nurses at both hospitals were asked to treat patients as normal. The notes of both attendances were later reviewed. 
H.R. Guly \& I.C.

Grant

RESULTS

Forty-six patients admitted that they had previously attended the other A\&E department for the same problem. One of these patients had also been treated in a third A\&E department in an adjoining health district and another patient had also been seen by two GPs for the same condition. Two sets of $A \& E$ notes from one hospital were unavailable although the register confirmed that the patients had attended.

Six patients (13\%) had attended the first A\&E department and had been booked in but had not waited to be seen by a doctor. In three cases this was because of perceived long waiting times but one patient did not wait because he had been told that he was likely to be asked to return the following morning to have a radiograph taken. Another patient left after being observed to steal bandages and one left without being seen after being violent and abusive to staff. One patient had signed his own discharge from the first hospital against medical advice.

The reasons stated for attending the second department are shown in Table 1.

In nine cases $(20 \%)$ the patient had been told to reattend by a GP or practice nurse. In one of these cases a patient with continuing symptoms following a head injury had been seen initially at the Royal Naval Hospital but was sent to Derriford Hospital for review because of the CT scanner and neurosurgical unit on that site, and in one case there was an administrative error which resulted in the patient reattending the wrong hospital. However, in the remaining seven cases, there was no evidence as

Table 1. Stated reasons for reattendance

\begin{tabular}{lc}
\hline Reason for reattendance & No. of patients \\
\hline Wanted a radiograph & $8^{*}$ \\
Concerns about condition & 23 \\
Wanted a second opinion & 5 \\
Concerns about a wound & 3 \\
Wanted crutches & 2 \\
Wanted analgesia & 1 \\
Wanted a bandage & 1 \\
More convenient & 1 \\
Brought by police against & \\
patient's will & 1 \\
Unknown & 1 \\
\hline * In one case it was more convenient to attend the other \\
department.
\end{tabular}

to why the patient was not sent back to the first hospital.

Seven patients who had been treated at the initial hospital $(17.5 \%)$ had an injury that was missed (Table 2). This excludes a patient who was told to return for radiograph in the morning. He attended the other hospital instead and was found to have a fracture. In addition, one patient had a false positive diagnosis (of a fractured lateral malleolus) corrected when he attended the other department with plaster problems.

Five patients $(11 \%)$ had a second set of radiographs taken which would not have been necessary had they reattended the original hospital where the first set would still be on file.

Twelve patients at the second visit were admitted or followed up in some way $(31 \%$ of patients, excluding those who had not waited to be seen by a doctor or who had signed their own discharge) (see Table 3).

One of the two A\&E departments had a policy that patients with minor injuries presenting at night would be treated symptomatically and asked to return the following morning for a radiograph. Two patients, on receiving this advice, went straight to the other hospital where they were seen and had radiographs taken. One patient having been told

Table 2. Diagnostic errors made at initial visit

\begin{tabular}{|c|c|}
\hline Diagnostic error & No. of patients \\
\hline Fracture & $\begin{array}{l}3 \text { (scapula, toe, spiral fracture } \\
\text { of tibia - all missed bacause } \\
\text { of failure to take radiographs) }\end{array}$ \\
\hline Foreign body in wound & $\begin{array}{l}2 \text { (missed because of failure to } \\
\text { take radiographs }-1 \\
\text { required no treatment) }\end{array}$ \\
\hline Tendon injury & 1 (closed Boutonniere deformity) \\
\hline
\end{tabular}

Table 3. Patients admitted or followed up at the second hospital (excluding those who had not waited to be seen by a doctor or who had signed their own discharge at the first hospital)

\begin{tabular}{ll}
\hline Outcome of attendence & \multicolumn{2}{c}{ No. of patients } \\
\hline Admitted & 1 (head injury and was \\
& \multicolumn{2}{c}{ admitted for observation) } \\
Fracture clinic follow-up & 2 \\
A\&E clinic follow-up & 8 \\
Physiotherapy follow-up & 1 \\
\hline
\end{tabular}


Patients who attend two A\&E departments that this was likely to be the advice given did not wait to see a doctor and went straight to the other hospital.

Three patients who had not had radiographs taken were told to return to the A\&E department if their symptoms persisted but chose to go to the other A\&E department instead and three patients had had follow-up appointments made but chose to go to the other department in the meantime.

\section{DISCUSSION}

Forty-six patients who reattended a different $A \& E$ department in 4 months is a small number of patients but is almost certainly an under-estimate as this only includes patients who volunteered that they had been treated at the other A\&E department. Patients were not specifically asked whether they had been treated elsewhere.

Patients expectations of healthcare continue to increase. Many patients have unrealistic expectations, and will continue to seek treatment in an attempt to meet these expectations. Some of the 'dual attenders' in this study had already had several correct opinions and adequate management, yet remained unsatisfied enough to seek further advice.

While some dual attendances may be the result of hospital-related reasons, such as missed diagnoses, many are not the fault of the A\&E department staff, but may be the result of patient-related factors, or poor availability of primary health care. ${ }^{3}$

The general public need to be educated. Careful advice at the initial visit, both in terms of what to expect and action to be taken in the event of these expectations not being met, should help to reduce inappropriate attendances. Patients should be strongly advised that for continuity of care and in order to avoid unnecessary additional investigations, including exposure to X-rays, they should return to the same department.

Similarly, a patient who subsequently sees his or her GP after an A\&E attendance should, if necessary, be referred back to the original hospital. Occasionally the GP may have a good reason for wishing the patient to be seen elsewhere, in which case that should be stated in the referral latter.

A proportion of patients, as a result of intoxication or other factors, sought further review after some degree of altercation in the first department. Abusive behaviour was not unusual and criticism was often implied. Such abuse of the system is difficult to prevent in areas offering open access to patients.

Eighteen per cent of patients had had diagnostic errors made at the original department and three claimed that they had had crutches or analgesia denied them at the original department. It is clearly important that doctors who make diagnostic or management errors are informed about them and that these errors are noted, acted on and audited. This will not occur if patients go back to a different department.

\section{CONCLUSION}

In cities where there is more than one A\&E department, there should be a mechanism whereby if a patient treated in one A\&E department later attends another, the first department is informed of the second visit. This is particularly important if any diagnostic or management error has been discovered. We also recommend that such departments should have common policies, for example policy about radiological examination as a means of avoiding some of the problems of dual attendances.

\section{ACKNOWLEDGEMENTS}

We would like to thank the reception, nursing and junior staff in both departments for identifying the patients and Mrs C. McBride for typing the paper.

\section{REFERENCES}

1. Moore R.S. (1987) The SOS patient: reasons for unexpected return to the accident and emergency department. British Journal of Accident and Emergency Medicine 2, 7-8.

2. O'Dwyer F. \& Bodiwala G.G. (1991) Unscheduled return visits by patients to the accident and emergency department. Archives of Emergency Medicine 8, 196-200.

3. Milner P., Beeby N. \& Nicholls J. (1991) Who should review the walking wounded? Reattendance at Accident and Emergency departments. Health Trends 23, 36-41. 